\title{
EDUCAÇÃO DE QUALIDADE NA ENFERMAGEM: FENÔMENO COMPLEXO E MULTIDIMENSIONAL
}

\author{
Dirce Stein Backes ${ }^{1}$, Claudia Zamberlan², Hedi Crecencia Heckler de Siqueira ${ }^{3}$, Marli Terezinha Stein Backes ${ }^{4}$, \\ Francisca Georgina Macêdo de Sousa ${ }^{5}$, Maria de Lurdes Lopes de Freitas Lomba ${ }^{6}$
}

\footnotetext{
${ }^{1}$ Doutora em Enfermagem. Docente do Curso de Enfermagem e do Mestrado Profissional em Materno Infantil da Universidade Franciscana (UFN). Santa Maria, Rio Grande do Sul, Brasil. E-mail: backesdirce@unifra.com.br

${ }^{2}$ Doutora em Enfermagem. Docente do Curso de Enfermagem e do Mestrado Profissional em Materno Infantil da UFN. Santa Maria, Rio Grande do Sul, Brasil. E-mail: claudiaz@unifra.br

${ }^{3}$ Doutora em Enfermagem. Docente do Programa de Pós Graduação em Enfermagem da Universidade Federal do Rio Grande. Rio Grande, Rio Grande do Sul, Brasil. E-mail: hedihs@terra.com.br

${ }^{4}$ Doutora em Enfermagem. Docente do Departamento de Enfermagem e do Programa de Pós-Graduação de Enfermagem da Universidade Federal de Santa Catarina. Florianópolis, Santa Catarina, Brasil. E-mail: marli.backes@ufsc.br

${ }^{5}$ Doutora em Enfermagem. Docente do Programa de Pós-Graduação de Enfermagem da Universidade Federal do Maranhão. São Luís, Maranhão, Brasil. E-mail: fgeorginasousa@hotmail.com

${ }^{6}$ Doutora em Ciências de Enfermagem. Professor do Curso de Licenciatura em Enfermagem e do Mestrado em Enfermagem de Saúde Infantil e Pediátrica na Escola Superior de Enfermagem de Coimbra. Coimbra, Portugal. E-mail: mlomba@esenfc.pt
}

\section{RESUMO}

Objetivo: refletir sobre a educação de qualidade na Enfermagem como fenômeno complexo e multidimensional.

Método: trata-se de um estudo teórico-reflexivo desenvolvido e sistematizado em cinco unidades de reflexão: ambientes de aprendizagem significativa; metodologias ativas; convivência em realidades distintas; Redes colaborativas; e, Abordagens complexas de intervenção.

Resultados: a análise das unidades de reflexão, à luz do pensamento complexo, demonstraram que a aprendizagem, na contemporaneidade, não pode mais ser concebida como processo pontual e linear. A educação de qualidade está relacionada à ampliação das interações e associações sistêmicas e à capacidade de fortalecer a interlocução com a realidade complexa e em constante mudança.

Conclusão: necessita-se transcender o paradigma da simplificação e considerar a complexidade intrínseca que se encontra no cerne da ciência. DESCRITORES: Educação em enfermagem. Educação superior. Dinâmica não linear. Qualidade.

\section{QUALITY NURSING EDUCATION: A COMPLEX AND MULTIDIMENSIONAL PHENOMENON}

\begin{abstract}
Objective: to reflect on quality Nursing education as a complex and multidimensional phenomenon.

Method: this is a theoretical-reflexive study developed and systematized into five units of reflection: Significant learning environments; active methodologies; Interactions in different realities; collaborative networks; and complex intervention approaches.

Results: based on complex thinking, an analysis of the units of reflection has shown that learning in the contemporaneity can no longer be conceived as a specific and linear process. Quality education is related to expanding systemic interactions and associations, and to the ability to strengthen its relation with a constantly changing complex reality.

Conclusion: it is necessary to transcend the simplification paradigm, taking into account the intrinsic complexity that lies at the heart of science.
\end{abstract}

DESCRIPTORS: Nursing education. Higher education. Non-linear dynamics. Quality. 


\section{EDUCACIÓN DE CALIDAD EN LA ENFERMERÍA: FENÓMENO COMPLEJO Y MULTIDIMENSIONAL}

\section{RESUMEN}

Objetivo: reflexionar sobre la educación de calidad en la Enfermería como fenómeno complejo y multidimensional.

Método: se trata de un estudio teórico-reflexivo desarrollado y sistematizado en cinco unidades de reflexión: ambientes de aprendizaje significativo, metodologías activas, convivencia en realidades distintas, redes colaborativas y abordajes complejos de intervención.

Resultados: el análisis de las unidades de reflexión a la luz del pensamiento complejo demostró que el aprendizaje, en la contemporaneidad, no puede ser concebido como un proceso puntual y lineal. La educación de calidad está relacionada con la ampliación de las interacciones, las asociaciones sistémicas y con la capacidad de fortalecer la interlocución con la realidad compleja y en constante cambio.

Conclusión: se necesita transcender el paradigma de la simplificación y considerar a la complejidad intrínseca que se encuentra en el cerne de la ciencia.

DESCRIPTORES: Educación en enfermería. Educación superior. Dinámica no lineal. Cualidad.

\section{INTRODUÇÃO}

A educação é um fenômeno complexo e multidimensional, incapaz de ser apreendida apenas pelo reconhecimento de variáveis quantitativas. A educação necessita desenvolver competências associativas capazes de interagir num mundo global, que valoriza, gradativamente, a capacidade crítico reflexiva e o caráter inovador e transformador dos problemas sociais. ${ }^{1-2}$ Entendida como fenômeno complexo, a educação de qualidade envolve a relação entre os recursos materiais e humanos, bem como os processos de ensino-aprendizagem, os currículos, as experiências, o compromisso e as atitudes dos docentes, as expectativas de aprendizagem, além do desempenho singular de cada aluno.

Em âmbito internacional, as discussões acadêmicas, na área da saúde, têm se voltado para a aprendizagem de alta qualidade. Discutem-se as implicações da mobilidade acadêmica internacional, da globalização do ensino, dos crescentes avanços tecnológicos, dos desafios relacionados às mudanças demográficas, do surgimento de novas demandas de saúde, das doenças crônicas, dentre outras. Nesse processo, as discussões se concentram, de forma recorrente, nas habilidades docentes necessárias à promoção da aprendizagem de qualidade..$^{1-2}$

Nacionalmente, as discussões se concentram nos atributos desejáveis ao processo educativo, tendo em vista a produção, a organização, a gestão e a disseminação de saberes e práticas fundamentais ao exercício da cidadania e da profissão. Para tanto, tem se investido em novas metodologias de ensino-aprendizagem, em práticas baseadas em evidências científicas, em tecnologias de inovação e em referenciais que sustentam a concepção sistêmica e o protagonismo social, tanto na formação acadêmica, quanto na educação continuada e permanente dos profissionais. No tocante às Diretrizes Curriculares Nacionais dos cursos da saúde, reconhece-se que es- tas têm provocado mudanças gradativas no modo de conceber e efetivar o processo ensino-aprendizagem, com crescente impacto às necessidades sociais. Em sua natureza, as Diretrizes Curriculares propõem a formação de profissionais Enfermeiros com visão generalista, humanista, crítica e reflexiva, para conhecerem e intervirem de forma responsável e competente no processo de cuidar em Enfermagem e saúde..$^{2-3}$

Na área de Enfermagem, importantes avanços podem ser constatados. Destacam-se os avanços relacionados às pesquisas acadêmicas, ao crescimento de programas de pós-graduação, à internacionalização do saber, à qualificação das metodologias de aprendizagem e o crescente impacto do cuidado de Enfermagem como prática social. Há evidências promissoras que sustentam os ambientes de aprendizagem significativa, as metodologias problematizadoras de resolução de problemas, os processos autocríticos e autorreflexivos baseados na formulação de boas perguntas ao invés de dar respostas prontas, dentre outros. ${ }^{3-4}$ Por outro lado, estudos demonstraram haver poucas mudanças qualitativas nas práticas de cuidado em saúde, bem como evidências científicas que sustentem o cuidado como sistema complexo e multidimensional. ${ }^{4-5}$

Para Morin, um dos autores do pensamento complexo, a educação perpassa, necessariamente, pela reforma do pensamento. Na visão do autor é preciso substituir um pensamento que reduz e fragmenta por um pensamento que distingue, une e integra, isto é, por um pensamento complexo, dinâmico e evolutivo. O autor reconhece, que não há problemas que não sejam complexos e, consequentemente, não há lugar para o saber simplificado. ${ }^{6-8}$

A construção do conhecimento, de modo geral, em especial o da Enfermagem, ocorre em fases evolutivas, as quais não se sucedem de forma linear e pontual, mas, processual e complementar. No período de Florence Nightingale, primeira fase, o foco do saber de Enfermagem centrou-se em responder 
ao questionamento "o que fazer?". Na segunda fase, tentando conquistar o domínio técnico, a Enfermagem procurou definir "como fazer?". A terceira fase teve como foco a investigação do "por que fazer?". Na quarta fase, buscou-se discutir "qual o saber próprio da enfermagem?" ${ }^{\prime 9} \mathrm{e}$, acrescentando uma quinta fase, questiona-se: como promover a educação de qualidade, na Enfermagem, como fenômeno complexo e multidimensional, tendo em vista a necessidade de apreender relações complexas que não nos foram ensinadas no modelo educacional mecanicista?

Com base no exposto, objetiva-se refletir sobre a educação de qualidade, na Enfermagem, como fenômeno complexo e multidimensional.

\section{EDUCAÇÃO DE QUALIDADE À LUZ DO PENSAMENTO COMPLEXO}

Trata-se de um estudo teórico-reflexivo que versa sobre a educação de qualidade à luz do pensamento complexo. Este estudo foi desenvolvido e sistematizado com base em cinco unidades de reflexão: Ambientes de aprendizagem significativa; Metodologias ativas; Convivência em realidades distintas; Redes colaborativas; e, Abordagens complexas de intervenção, as quais demonstraram que a aprendizagem de qualidade não pode mais ser concebida como processo pontual e linear. A promoção da qualidade implica na ampliação das interações e associações sistêmicas, bem como na capacidade de fortalecer a interlocução com a realidade complexa e em constante mudança.

A realidade social, como já mencionado, está em constante evolução e transformação. Praticamente, todas as pessoas têm acesso às mudanças quase que imediatamente. Logo, advém o questionamento recorrente: que competências e habilidades são necessárias para navegar por este oceano de incertezas? Que competências e habilidades necessitam ser desenvolvidas para manter a integração dinâmica e não "naufragar" entre as possíveis correntezas da travessia? Que habilidades e competências são necessárias para lidar com os novos fenômenos sociais que haverão de surgir? Como fazer frente às realidades sociais e de saúde, as quais requerem respostas cada vez mais complexas e multidimensionais?

Desenvolver a aprendizagem para um mundo em mudança, a um ritmo sem precedentes implica, a priori, quebrar as barreiras disciplinares, de modo que a aprendizagem sistêmica prevaleça sobre o saber linear e fragmentado; a multidimensionalidade sobre as causalidades determinísticas; a horizontalidade sobre as práticas verticalizadas; e, a complexidade sobre as abordagens simplificadoras. Os estudantes precisam adquirir, gradativamente, habilidades para pesquisar, analisar criticamente e questionar o incerto e o aleatório, porque, por definição, o novo não é previsível. ${ }^{10}$

\section{Ambientes de aprendizagem significativa}

Na lógica do pensamento complexo é preciso ultrapassar o ensino repetitivo e/ou transmissivo, isto é, a relação sujeito-objeto. O desafio da aprendizagem não é mais a aquisição verticalizada do conhecimento, mas fazer com que o aluno seja capaz de dar significado às coisas, a partir de sua compreensão ampliada e contextualizada. Sob esse enfoque, o maior desafio dos docentes é criar um ensino que atenda a uma nova forma de aprender, tendo em vista que o acúmulo de informações disponíveis exigem discernimento para escolher os conteúdos relevantes que, interconectados com o entorno, podem gerar novos conhecimentos significativos. ${ }^{6}$

A aprendizagem significativa, portanto, se concentra em valorizar o conhecimento prévio do aluno. Novos conhecimentos são adquiridos e ampliados na medida em que o aluno consegue associá-los ao conhecimento prévio. Para tanto, os ambientes de aprendizagem precisam ser agregadores e instigadores, a fim de que o estudante encontre significado nas novas informações e para que consiga conectar estas mesmas informações com a multidimensionalidade de fenômenos que o cercam. ${ }^{11}$

É preciso que se considere que a informação prévia já se constitui, na maioria das vezes, em significado para o aluno e que o influencie na construção de novas aprendizagens, as quais são incorporadas a partir das conexões com os diferentes saberes e realidades sociais. Nessa direção, a aprendizagem significativa pode ser definida como um processo pelo qual a nova informação se interconecta com a estrutura de conhecimento já existente. Cabe ao docente valorizar e potencializar o conhecimento prévio para construir as estruturas mentais que permitem descobrir e ampliar os novos conceitos. ${ }^{10}$

Possibilita-se, por meio da aprendizagem significativa, a participação ativa do aluno na construção do conhecimento. Nessa lógica, o aluno passa da concepção receptora e ação interlocutora. O conhecimento deixa de ser objeto de domínio do professor e passa a ser uma construção dialógica e de significado para ambos os envolvidos. Assim, o estudante torna-se sujeito ativo e reflexivo e o professor assumirá a posição de mediador e instigador do processo ensino-aprendizagem. ${ }^{11}$ 


\section{Metodologias ativas}

A aprendizagem significativa possibilita a interlocução ativa e autônoma do aluno na construção do conhecimento, além de possibilitar uma análise crítica da realidade. É importante que esse processo amplie as relações e associações sistêmicas. Nesse sentido, as metodologias ativas contribuem para a apreensão crítico-reflexiva das informações processadas, as quais requerem a participação e a interação do aluno com as ferramentas disponibilizadas. ${ }^{11}$

As metodologias ativas estão relacionadas às abordagens interativas adotadas no desenvolvimento do processo de ensino-aprendizagem. Estas podem estar interconectadas a experiências reais e são utilizadas, mais especificamente, quando se intenta contribuir para a aprendizagem baseada na resolução de problemas. Nessa abordagem, o estudante precisa apreender e compreender os fenômenos de forma abrangente e multidimensional, bem como propor possíveis estratégias de intervenção por meio do processo de ação-reflexão-ação. ${ }^{12}$

A problematização, privilegiada nessa metodologia, visa estimular o estudante a pensar, a refletir, a criar, a indagar-se e a (re)significar continuamente as suas descobertas. A problematização teórico-prática, como estratégia de ensino-aprendizagem, tem a possibilidade de proporcionar a conexão com as informações e a produção de conhecimento ampliado e contextualizado. Pela ampliação das possibilidades interativas e associativas, as metodologias ativas se constituem em estratégia de significado para o aluno desenvolver processos autocríticos e que estejam em consonância com as necessidades emergentes. Essa perspectiva é coerente com uma das características do pensamento complexo, que é a de um pensamento dialético, dinâmico e evolutivo, no qual nada se repete com as mesmas características, isto é, que sinaliza o caráter evolutivo do processo ensino-aprendizagem. ${ }^{11-12}$

A reforma do processo ensino-aprendizagem perpassa, necessariamente, pela reforma do pensamento. Desenvolver um pensamento que propicie a religação dos saberes, numa relação dialógica e de complementaridade é, portanto, o passo inicial. A função do docente não se limita, nessa relação, ao desenvolvimento de competências técnico-científicas, mas à capacidade de promover processos interativos e integradores, a partir da dinâmica existencial. ${ }^{13}$

\section{Vivência em realidades distintas}

A vivência em realidades distintas, além de possibilitar a ampliação do objeto pela compreensão complexa e multidimensional do fenômeno, permite ao aluno fazer novas e diferentes perguntas, ao invés de treinar boas respostas. Da mesma forma, desperta o aluno para um mundo em contínua transformação, para o qual dificilmente terá respostas prontas. Com base nessa relação dialógica, a aprendizagem ocorre quando o aluno, inserido em um contexto social/ global distinto, apreende o objeto de forma sistêmica e é capaz de realizar uma autocrítica. ${ }^{13} \mathrm{~A}$ aprendizagem significativa ocorre, nessa relação, quando o aluno consegue, a partir do conhecimento prévio, instigar-se e, por vezes, provocar uma desordem pessoal. Tal desordem, na lógica do pensamento complexo, abre a possibilidade para uma nova ordem, ou seja, para um novo aprendizado, mais significativo, mais complexo e de maior qualidade..$^{6-7}$

É importante, também, que sejam desenvolvidas habilidades comunicativas, além de competências sociais e políticas, a partir da inserção do estudante em ambientes complexos. Esse reconhecimento social da prática, para além das manifestações técnicas, sugere que o aluno compreenda as desarticuladas dimensões implícitas no papel do enfermeiro, sobretudo, em contextos adversos e de aparente incerteza. ${ }^{9}$ Nessa lógica, o professor assume uma postura de mediação, isto é, passa a oferecer ferramentas que auxiliam o aluno a interpretar e a analisar o entorno social de forma abrangente e multidimensional. A aprendizagem significativa pode ser caracterizada, nessa perspectiva, como a aquisição de conhecimento significativo em uma realidade concreta, na qual o próprio aluno, sob a mediação do professor, precisa desenvolver habilidades interativas e promover as devidas conexões para apreender os fenômenos sociais de forma ampliada, contextualizada e global. ${ }^{13}$

\section{Redes colaborativas}

O contexto atual, motivado pela Web, hipermídias, redes colaborativas entre outros recursos, sugere novos estilos sociais e de convivência, além da (re)significação do modus operandi dos profissionais, em geral. As possibilidades de informação e comunicação, essencialmente móveis, acenam para perspectivas dinâmicas, interativas e criativas na aprendizagem. Nessa plataforma interativa, o ser humano pode transitar por todo planeta sem mesmo sair fisicamente do seu lugar. A sociedade deixa de ser local e passa a ser global. Sob esse enfoque, as tecnologias de informação e comunicação permitem a interatividade da sociedade com potencial para mudar a cultura e a divulgação das informações, em geral, num processo irreversível. ${ }^{14}$ 
Com o advento das novas tecnologias de informação e comunicação, não há como permanecer alheio e/ou indiferente às mudanças que se apresentam na educação, tendo em vista que estas transcendem os limites da dimensão espacial, temporal, cultural e curricular, além de proporcionar interfaces entre os diferentes atores sociais. Na era da interconectividade, na qual sobressaem interação/interatividade é importante que se estimule a convivência com a diversidade de ideias, o diálogo, a cidadania e o trabalho de produção colaborativa. Para tanto, é preciso que sejam criadas propostas pedagógicas que incorporem as potencialidades mediadoras que contemplem as tecnologias de informação e comunicação, além da flexibilização dos currículos, criando espaços e tempos de atuação docente e discente compatíveis com as demandas atuais. ${ }^{15}$

O mundo é, portanto, uma rede em conexões, interconexões, movimento, fluxo de energia e inter-relações, isto é, em constante processo de mudança e transformação. Nessa lógica, as redes colaborativas se configuram como estruturas dinâmicas e se caracterizam como cadeias associativas entre indivíduos ou grupos, baseadas em processos dialógicos de aprendizagem, as quais são mais adaptáveis, dinâmicas e, consequentemente, mais flexíveis e adaptáveis às continuas mudanças. Nesse movimento paradoxal, altamente dinâmico e interativo, a educação necessita repensar a sua função social e, gradativamente, fomentar as redes colaborativas em âmbito local e global. ${ }^{16}$

\section{Abordagens complexas de intervenção}

O processo de aprendizagem não se sustenta, num momento de transição paradigmática, em abordagens simplificadoras, nas quais vigoram relações verticalizadas e hegemônicas, mas em uma visão complexa, que se fundamenta na superação da visão fragmentada, linear e reducionista do universo. No processo de aprendizagem, o professor tende, frequentemente, a reduzir e/ou facilitar as ideias aos estudantes, a fim de torná-las mais simples de serem decodificadas. Esse modo de proceder supõe que os fenômenos são simples e fáceis de serem resolvidos. Na lógica do pensamento complexo, a aprendizagem se sustenta em abordagens que transcendem o olhar simplificado e que buscam alcançam a singularidade e a multidimensionalidade dos diferentes atores e componentes envolvidos no processo. ${ }^{6}$

Modelos tradicionais de ensino-aprendizagem são, portanto, na atualidade, cada vez mais questionados e provocados à luz de novos referenciais que se sustentam no pensamento complexo. Esse é um dos referenciais que possibilita a construção de saberes multidimensionais pelo seu caráter circular e interativo que vai além da somatória de conteúdos programáticos. O pensamento complexo visa à superação da consciência ingênua para o alcance de uma consciência crítica, capaz de apreender o mundo como rede de múltiplas relações, as quais se encontram em constante transformação. O professor como mediador do processo de aprendizagem necessita, nessa lógica, estar apto para integrar, religar e instigar a construção do conhecimento no campo singular e multidimensional, além de conciliar as instâncias individuais e coletivas. Assim, tanto o professor quanto o aluno serão sujeitos da aprendizagem significativa, emancipadora e transformadora. ${ }^{6}$

Para desenvolver a educação de qualidade na Enfermagem e, consequentemente, qualificar o cuidado em saúde é preciso que os Enfermeiros pensem e reflitam criticamente sobre a sua prática, a partir da resolução de problemas complexos. Para tanto é indispensável que os Enfermeiros possibilitem, no processo formativo, ferramentas de negociação e processos dialógico de ação-reflexão-ação para a compreensão ampliada e contextualizada dos fenômenos sociais e de saúde. ${ }^{11}$

A educação de qualidade, na perspectiva da complexidade, é produto e produtora da reforma do pensamento. Requer-se, para tanto, um ensino baseado na problematização e na contextualização que são a base das metodologias inovadoras e instigadoras. Um ensino que propicie a religação dos saberes e a capacidade de compreender, integrar, agregar e, sobretudo, superar a visão fragmentada, linear e reducionista dos fenômenos sociais. É preciso, portanto, substituir o pensamento linear que separa e fragmenta por um pensamento complexo e multidimensional, capaz de religar e sistematizar os conhecimentos, muito mais do que reproduzi-los por meio de conteúdos programáticos. ${ }^{6,17}$

\section{CONCLUSÃO}

A educação de qualidade está relacionada à ampliação das interações e associações sistêmicas e à capacidade de fortalecer a interlocução com a realidade complexa e em constante mudança. É preciso, gradativamente, transcender o paradigma da simplificação e considerar a complexidade intrínseca que se encontra no cerne da ciência.

O pensamento complexo, iluminador da educação de qualidade, transcende a soberania da ordem instituída e concebe a relação dialógica entre a ordem, a desordem e a organização. Nessa 
relação, o professor assume o papel de mediador da aprendizagem, o que implica em valorizar e potencializar as singularidades humanas, além de apreender os fenômenos de forma multidimensional. É preciso que o professor seja capaz de transcender velhos paradigmas educacionais pela problematização de sua prática e a partir de novos questionamentos.

O pensamento complexo possibilita, por natureza, a capacidade reflexiva do sujeito sobre si e sobre o seu estar e agir no mundo. Nessa direção, a reflexibilidade permite a transposição de limites pessoais impostos pelo pensamento instituído e, respectivamente, a adoção de ações comprometidas. A educação de qualidade, à luz do pensamento complexo, remete à prática concebida pela relação intrincada de fios que se entrecruzam numa rede plural e multidimensional de saberes. Nesse sentido, as disciplinas e conteúdos especializados continuarão sendo importantes, mas, sua relevância está na capacidade de interligá-los e interconectá-los com outros saberes.

Para o alcance desse processo é preciso transcender o paradigma da simplificação, caracterizado por princípios de disjunção, de redução e de fragmentação e, gradativamente, considerar a complexidade dos fenômenos sociais. Permanece, portanto, o desafio da educação de qualidade à luz de referenciais complexos, bem como a adoção de estratégias que permitam legitimar e incorporar o conhecimento científico ao processo de cuidado de Enfermagem, na prática.

\section{REFERÊNCIAS}

1. Coutinho C, Lisbôa E. Sociedade da informação, do conhecimento e da aprendizagem: desafios para educação no século XXI. Rev Educ. 2011; 18(1):5-22.

2. Ministério da Educação (BR). Educação Brasileira: indicadores e desafios. Fórum Nacional de Educação. Brasília (DF): Ministério da Educação; 2013.

3. Conselho Nacional de Educação (BR), Câmara de Educação Superior. Diretrizes Curriculares Nacionais do curso de graduação em enfermagem. Diário Oficial da República Federativa da União. Brasília (DF): Ministério da Educação; 2001.

4. Silva RS. Pós-Graduação e a pesquisa em Enfermagem na América Latina: Avanços e desafios. Rev Cid [Internet]. 2015 [cited 2017 Mai 23]; 6(2):1019-21. Available from: http://dx.doi.org/10.15649/cuidarte. $\underline{\mathrm{v} 6 \mathrm{i} 2.307}$
5. Pereira WR. Produção de conhecimento em enfermagem: transposição e repercussões no ensino de graduação. Rev Bras Enferm [Internet]. 2013 Sep [cited 2016 Oct 11]; 66(Spe):111-8. Available from: http:/ / www.scielo.br/scielo.php?script=sci_ arttext\&pid=S0034-71672013000700015\&lng=en

6. Morin E. Sete saberes necessários a educação do futuro. Rio de Janeiro (RJ): Cortês; 2016.

7. Morin E. Ciência com consciência. Rio de Janeiro (RJ): Bertrand Brasil; 2017.

8. Dürks DB, Silva SP. Ambivalência, complexidade e conhecimento: Bauman e Morin. Controvérsia [Internet]. 2014 [cited 2017 May 23]; 10(1):35-43. Available from: http://revistas.unisinos.br/index. $\mathrm{php} /$ controversia/article/viewFile/9851/4742

9. Backes DS, Backes MTS, Erdmann AL, Büscher A. Do padrão nightingaleano de enfermagem ao sistema social luhmanniano: estudo teórico. Rev Bras Enferm [Internet]. 2013 Aug [cited 2016 Oct 11]; 66(4):599-602. Available from: http://dx.doi.org/10.1590/S003471672013000400020 .

10. Giusta AS. Concepções de aprendizagem e práticas pedagógicas. Educ Rev [Internet]. 2013 Mar [cited 2017 Mai 22]; 29(1):20-36 Available from: https:/ /dx.doi. org/10.1590/S0102-46982013000100003

11. Borges TS, Alencar G. Metodologias ativas na promoção da formação crítica do estudante: o uso das metodologias ativas como recurso didático na formação crítica do estudante do ensino superior. Cairu em Revista [Internet]. 2014 [cited 2017 May 23]; 3(4): 119-43. Available from: http://www. cairu.br/revista/arquivos/artigos/2014_2/08\%20 METODOLOGIAS \% 20ATIVAS \% 20NA \% 20 PROMOCAO \% 20DA \% 20FORMACAO \% 20 CRITICA \% 20DO\% 20ESTUDANTE.pdf

12. Vernaschi LV. Espiral construtivista: uma metodologia ativa de ensino-aprendizagem. Interface (Botucatu) [Internet]. 2017 Jun [cited 2017 May 24]; 21(61):421-34. Available from: http://dx.doi.org/10.1590/180757622016.0316.

13. Sabzevari S, Abbaszade A, Borhani, F. The assessment methods and learning approaches in nursings students of kerman university of medical sciences in Iran. Creative Education. 2013; 4(2):160-4.

14. Backes DS, Backes MTS, Lunardi VL, Erdmann AL, Büscher A. Internacionalização como desafio ao impacto da globalização: contribuições da enfermagem. Rev Esc Enferm USP [Internet]. 2014 Oct [cited 2017 
May 24]; 48(5):772-7. Available from: http:/ /dx.doi. org/10.1590/S0080-623420140000500001.

15. Gama LN, Tavares CMM. Education and media: contemporary implications in the academic routine. Texto Contexto Enferm [Internet]. 2015 June [cited 2017 May 24]; 24(2):593-9. Available from: http:/ / dx.doi.org/10.1590/0104-07072015002052014.

16. Brewster L, Mountain G, Wessels B, Kelly C, Hawley
M. Factors affecting front line staff acceptance of telehealth technologies: a mixed-method systematic review. J Adv Nurs [Internet]. 2014 Jan [cited 2017 May 24]; 70(1):21-33. Available from: http://dx.doi. org/1010.1111/jan.12196.

17. Castro EA. Pensamento complexo educação e direitos humanos. Periódico Científico Projeção e Docência. 2014; 5(2):70-80. 